\title{
Sobre la validez legal del texto traducido: análisis de un End User License Agreement y su traducción al español
}

\author{
José RAMÓN CALVO-FERRER \\ Universidad de Alicante \\ jr.calvo@ua.es
}

Recibido: 24 de febrero de 2014

Aceptado: 14 de marzo de 2014

\section{RESUMEN}

El presente artículo tiene como objeto analizar los distintos factores que conforman la traducción de un End User License Agreement al español y examinar las peculiaridades lingüísticas que se originan durante dicho proceso y que permiten al lector identificar el texto resultante como un producto extraño en lugar de como un texto surgido de forma original en el ordenamiento jurídico español y que ha de ser observado como tal por sus destinatarios. Con ello en mente, se analizan aquellos contenidos que pudieran contravenir la normativa vigente y se debate acerca de la validez del End User License Agreement como instrumento jurídico en su forma traducida.

Palabras clave: traducción de textos jurídicos, contrato de licencia, ordenamiento jurídico español, condicionantes de traducción.

Remarks on the validity of the translated text: Analysis of an End User License Agreement and its translation into Spanish

\begin{abstract}
This article aims to analyse the different factors that shape the translation of an End User Agreement into Spanish and to scrutinise the linguistic peculiarities which arise during such process and which allow the reader to identify the resulting document as an alien product rather than an original legal text that needs to be observed by its end users. Bearing such issues in mind, both the contents that may violate the current Spanish legislation and the validity of the End User Agreement as a legal instrument within the Spanish legal system are subsequently analysed.
\end{abstract}

Keywords: translation of legal texts, End User License Agreement, Spanish legal system, translation constraints.

Sumario: 1. Introducción. 2. Los condicionantes de traducción. 3. Los End User License Agreements o Contratos de licencia de usuario final. 4. Consideraciones legales sobre los 
End User License Agreements y sus equivalentes Contratos de licencia de usuario final. 5. Consideraciones discursivas sobre los End User License Agreements y sus equivalentes Contratos de licencia de usuario final. 6. Conclusiones.

\section{Introducción}

No son pocas las ocasiones en las que el ser humano lleva a cabo acciones más o menos complejas de forma mecánica, sin prestar excesiva atención a lo que tiene entre manos. Este modo de proceder, que los ingleses denominan second nature y que engloba conductas tan repetitivas como cambiar de marcha mientras se conduce o batir un huevo frente a la televisión, parece ponerse de relieve cada vez que se acepta de forma sistemática los múltiples contratos de licencia de usuario final a los que se da consentimiento para poder instalar programas informáticos en el ordenador. Tales contratos, presentes en la mayoría de aplicaciones de software, ya sean libres o de pago, contienen un gran número de cláusulas y condiciones que pasan desapercibidas para la mayoría de los usuarios, que presuponen la corrección de lo escrito o se encuentran impacientes quizá por empezar a utilizar el software en cuestión.

El estudio que sigue es una aproximación descriptiva a los condicionantes que repercuten en el aspecto final de la traducción de los End User License Agreements a nuestro idioma y su adecuación con el resto de contratos en el marco del lenguaje jurídico-administrativo español. Por medio de un análisis exhaustivo de los procedimientos empleados en la traducción de uno de estos textos al español, se intentará llevar a cabo un estudio tanto de aquellos factores que inciden en el aspecto final del texto como del modo en que se presentan en el mismo.

Para ello, se ofrecerá en primer lugar una aproximación al objeto de estudio, la noción de condicionante de traducción y el End User License Agreement, analizando de forma sucinta sus características y peculiaridades y observando aquellos aspectos intrínsecos a esta modalidad de contrato que pudieran dificultar su trasvase terminológico y conceptual al castellano. Posteriormente, una vez efectuado este breve análisis, se cotejará el EULA aparecido durante el proceso de instalación del programa Adobe Reader 8.0 con su traducción en lengua castellana con el fin de evaluar la presencia de aquellos condicionantes externos que hayan derivado en la aparición de determinadas características textuales y, en su caso, realizar propuestas para la eventual mejora del texto traducido. Del mismo modo, se presentarán distintas propuestas para la mayor adecuación del texto traducido a la tipología textual jurídico-administrativa española, resolviendo de forma crítica los posibles conflictos textuales que de este proceso se pudieran derivar, acompañadas de unas consideraciones personales evaluadoras del resultado investigador alcanzado.

\section{Los condicionantes de traducción}

Atendiendo a la explicación dada por Montero Martínez et al. (2001: 283), los condicionantes de traducción son aquellos elementos que someten al «sujeto que 
interviene en el proceso traductor» y que provocan, entre otros, el uso del «calco sintáctico como técnica traductológica de unidades fraseológicas tales como las unidades léxicas».

Los condicionantes de traducción pueden ser externos al texto, como en el caso de la formación del profesional traductor, de la premura del encargo de traducción o de la aparición del original junto al texto traducido, o bien internos al mismo, como en el caso de la ordenación de los párrafos o de la distinta terminología empleada. Resulta a todas luces evidente que tanto un tipo de condicionante como otro ponen a prueba la capacidad del traductor para alcanzar una traducción válida de acuerdo con los estándares de corrección vigentes en el momento de la realización de la misma.

\section{Los End User License Agreements o Contratos de licencia de usuario final}

Un tipo textual con importantes condicionantes externos e internos es el End User License Agreement, referido en español como Contrato de licencia de usuario final o $C L U F$. De acuerdo con la definición proporcionada por López-Tarruella Martínez (2006: 89), un EULA es una licencia de utilización de software estándar unipersonal, esto es, con un solo usuario autorizado, en cuyo clausulado «el proveedor informático puede establecer las condiciones y limitar el uso que el usuario puede hacer del software». Como ya se ha apuntado en la introducción, éste es un tipo de contrato que establece las normas que rigen el uso de software o, dicho con otras palabras, el instrumento legal que permite su uso a terceros.

La licencia de software tiene una doble función: por una parte, otorga al usuario una serie de derechos sobre el mismo, mientras que por otra impone ciertas restricciones sobre su uso. Entre las restricciones más significativas, encontramos la expresada a través del artículo 99 de la Ley de la Propiedad Intelectual española: «cuando se produzca cesión del derecho de uso de un programa de ordenador se entenderá, salvo prueba en contrario, que dicha cesión tiene carácter no exclusivo e intransferible, presumiéndose, asimismo, que lo es para satisfacer únicamente las necesidades del usuario».

Tal y como se ha mencionado anteriormente, existe una diferenciación entre software gratuito y software de pago o, según la terminología utilizada con más frecuencia, software libre y propietario. Según Malcolm Bain et al. (2004: 355), dado que la difusión del software propietario se fundamenta en la obtención del mayor rendimiento económico posible, sus licencias poseen «un contenido y alcance muy restrictivo de las facultades que sobre el software se conceden al usuario, así como muy protector de los derechos en exclusiva del autor», mientras que las licencias de software libre no se conciben para proteger la explotación del producto, sino para «conceder y asegurar a los usuarios las libertades de uso, modificación y redistribución (con o sin modificaciones) sobre el software».

Del mismo modo, un EULA se puede presentar de distintas formas. Por una parte, los llamados shink-wrap contracts son aquellas licencias de software que se presentan en el interior de la caja del programa, habitualmente en una funda trans- 
parente. Dado su formato impreso, este tipo de licencias se encuentran asociadas a los programas de software propietario. Por otra parte, son frecuentes las licencias en forma de cuadros de texto o click-wraps que se despliegan en la pantalla del ordenador durante el proceso de instalación del software en cuestión, típicamente de carácter libre.

El CLUF, surgido como consecuencia de la traducción al castellano de los EULA incluidos en los programas de software desarrollados en el extranjero, da origen a una tipología textual con unas características propias generalmente originadas por unos fuertes condicionantes de traducción, que con cierta frecuencia resultan en producciones lingüísticas que contravienen las convenciones formales y estructurales del marco jurídico en el que se engloban. El siguiente apartado aborda el análisis de varios de los condicionantes presentes en la traducción de los EULA al castellano y las consecuencias textuales que de ellos se derivan.

\section{Consideraciones legales sobre los End User License Agreements y sus equivalentes Contratos de licencia de usuario final}

Tal y como se ha indicado anteriormente, el contrato de licencia de software o CLUF no es un documento de gran tradición dentro del ordenamiento jurídico español. Su origen se debe en gran parte a la traducción de los EULA, redactados mayoritariamente en lengua inglesa como reflejo de los mercados en los que inicialmente vieron la luz. Bien es cierto que el mercado español y latinoamericano cuenta con numerosas empresas dedicadas al desarrollo de software, pero en las licencias de uso redactadas originalmente en castellano sometidas a análisis se observan claras similitudes con los CLUF traducidos de la lengua inglesa.

Para entender todo ello, se antoja conveniente indagar en el surgimiento del propio software. Inicialmente, IBM, dominadora absoluta del mercado informático, no comercializaba los programas de software de forma independiente, sino que los distribuía junto con el hardware, por lo que no existía tecnología capaz de realizar copias de los mismos ni preocupación alguna por su protección intelectual, si bien los consumidores se veían obligados a pagar por programas a los que probablemente no darían uso si deseaban adquirir una computadora (Baase, 1974).

Este vínculo se rompió en 1969 cuando, a causa de la legislación antimonopolio estadounidense, el gigante azul se vio obligado a separar sus negocios de hardware y software (Bain et. al., 2004: 51), abriendo el mercado a posibles competidores. Hasta ese momento, los contratos realizados entre proveedor y usuarios habían sido de compraventa, pero cuando los programas de ordenador empezaron a estandarizarse y comercializarse en soportes movibles, la copia se generalizó, y con ello se hubo de crear los EULA para que protegieran los derechos de autor a través de cesiones del derecho de uso de los mismos.

Es por ello que los CLUF poseen características propias muy marcadas como consecuencia de este modus nascendi, caracterizados principalmente por un gran apego tanto en la forma como en el contenido a su EULA original. De hecho, el término que les da nombre no se encuentra del todo acomodado en lengua castellana: 
mientas que se observa una tendencia creciente a la utilización del acrónimo CLUF, Contrato de Licencia de Usuario Final, traducción literal de los significantes ingleses, existe un alto porcentaje de referencias a este instrumento jurídico bajo su acrónimo inglés. Por otra parte, aquellas referencias efectuadas en castellano tampoco logran ponerse de acuerdo en la terminología a emplear; utilizando el motor de búsqueda Google encontramos aceptación tanto del término acuerdo de licencia de usuario final (327.000 incidencias) como del término contrato de licencia de usuario final (583.000 incidencias).

Por otra parte, Gómez Perals (1999: 83) indica la mayor idoneidad del término «cesión de uso» de los programas de ordenador. Según el citado autor, bajo la perspectiva del Derecho Comparado, en el ordenamiento jurídico español, en la Ley de la Propiedad Intelectual (Título V), «encontramos los términos relativos a las diferentes clases de transmisión; concretamente, cesión en exclusiva (48 y 49, entre otros), cesionario no exclusivo (50.1), autorizaciones no exclusivas (50.2)», mientras que los Estados Unidos ofrecen los conceptos de «transfer (cesión global o venta de la propiedad), de assignment (venta de ciertos derechos) y de license (licencia, autorización o concesión)».

Nos encontramos, por tanto, ante un instrumento cuya función resulta evidente pero cuya variedad terminológica es, en su ámbito de uso, del todo confusa. Dicha función, como se puede comprobar al inicio del EULA analizado (junto con su correspondiente CLUF) es claramente vinculante:

«YOU AGREE THAT THIS AGREEMENT IS ENFORCEABLE LIKE ANY WRITTEN NEGOTIATED AGREEMENT SIGNED BY YOU. THIS AGREEMENT IS ENFORCEABLE AGAINST YOU AND ANY LEGAL ENTITY THAT OBTAINED THE SOFTWARE AND ON WHOSE BEHALF IT IS USED. IF YOU DO NOT AGREE, DO NOT USE THIS SOFTWARE». «USTED ACEPTA QUE ESTE CONTRATO ES EXIGIBLE COMO CUALQUIER CONTRATO POR ESCRITO NEGOCIADO Y FIRMADO POR USTED. ESTE CONTRATO ES EXIGIBLE CONTRA VD. Y CONTRA CUALQUIER ENTIDAD QUE HAYA OBTENIDO EL SOFTWARE Y EN CUYO NOMBRE SE USE. SI USTED NO ESTÁ DE ACUERDO, NO UTILICE ESTE SOFTWARE».

No obstante, surgido este dualismo, resulta lógico plantearse qué licencia prevalecería en caso de conflicto entre el EULA y el CLUF. Bain et al. (2004: 450) son claros al respecto: si el autor del software aplica una licencia traducida a un programa, dicha traducción prevalece sobre la licencia original, salvo que exista alguna cláusula que disponga lo contrario. En el caso que nos ocupa, el CLUF analizado prevalecería sobre el EULA original, ya que no existe ninguna disposición al contrario a lo largo de todo el documento.

Por otra parte, como ya hemos mencionado, el EULA es aquel documento al que el usuario ha de dar su consentimiento para poder utilizar el software al que acompaña. Sin embargo, existen casos en los que el usuario puede aceptar las condiciones del mismo de forma involuntaria o inconsciente. Por ejemplo, como ya se ha visto, los shink-wrap contracts son aquellas licencias que se incluyen en el interior 
de una funda de plástico transparente que alberga asimismo al programa en cuestión. En numerosas ocasiones, la rotura de este plástico o la apertura de la caja que lo contiene implican la aceptación de las condiciones del EULA. Resulta evidente que en aquellos casos en los que la totalidad de la licencia de uso se encuentra parcialmente oculta o doblada, el usuario ha de romper su envoltorio para poder leerla en su totalidad, con la consecuente paradoja legal que ello supone.

Del mismo modo, en la mayoría de casos en los que la licencia aparece por medio de un cuadro de texto durante la instalación del programa concreto se pueden aceptar las condiciones de la misma sin haberlas leído en su totalidad. Lo que es más grave, en ocasiones se realiza de forma mecánica e inintencionada, ya que el botón de aceptación suele aparecer premarcado o bien ocupa la misma posición del botón pulsado inmediatamente antes.

De acuerdo con la Ley sobre Condiciones Generales de la Contratación, aplicable a este tipo de licencias por estar constituidas por condiciones generales redactadas unilateralmente por el proveedor de software, es necesario que en las licencias se den una serie de condiciones para que éstas puedan considerarse contratos y sean exigibles como tal. Entre ellas, y al respecto de lo que nos ocupa, destaca la exigencia al proveedor de posibilitar que el usuario tenga la oportunidad de conocer tales condiciones y aceptarlas. Por tanto, las licencias click-wrap y shrink-wrap no tendrán validez alguna en la medida que no sean capaces de probar que el usuario ha podido conocer y aceptar libremente las condiciones de la licencia y, por tanto, no podrán ser exigibles en supuestos casos de incumplimiento.

Otra de las cuestiones que suscita no poca controversia entre proveedores y usuarios es el aparente carácter abusivo de ciertas cláusulas contenidas en el contrato de licencia. En la mayoría de las ocasiones, el proveedor no se responsabiliza de los daños causados por un software defectuoso y, en otras, obliga al consumidor a aceptar servicios no solicitados de forma expresa o bien instala software innecesario para el correcto funcionamiento del programa deseado. A este tipo de comportamientos abusivos añade Bain et. al. (2004: 309) el hecho de que, en caso de litigio sobre la licencia, se impongan tribunales distintos a los del domicilio del usuario para su resolución o que la licencia se someta a un derecho ajeno a las partes, como resulta el presente caso, en el que Adobe, un proveedor de software estadounidense, impone a los usuarios españoles acudir a la jurisdicción inglesa para la resolución de los eventuales conflictos. De acuerdo con la Ley General para la Defensa de los Consumidores, este tipo de cláusulas, estipuladas unilateralmente y sin mediar ningún tipo de negociación entre las partes, podrían declararse nulas en caso de litigio, ya que suponen un serio perjuicio para el consumidor y causan un serio desequilibrio entre los derechos y obligaciones de una y otra parte.

De hecho, distintos tribunales han atendido a reclamaciones interpuestas por los usuarios: un tribunal de Estados Unidos estableció en 2009 que la adquisición de un software bajo un End User License Agreement es una compraventa y no una licencia, motivo por el cual la first sale doctrine (o agotamiento del derecho) no resulta aplicable y permite, entre otras cosas, la reventa del mismo por parte del usuario, tal como refrenda la sentencia de 2012 del Tribunal del Justicia de la Unión Europea, «An author of software cannot oppose the resale of his 'used' licences allowing the 
use of his programs downloaded from the internet», que estipula que el derecho exclusivo de distribución de un software cubierto por una licencia de uso se agota en su primera venta, independientemente, por tanto, de cuanto pueda expresar su correspondiente End User License Agreement.

Pese a todo, podemos observar que gran parte de las versiones en español de las licencias de software mantienen las clausulas tal como se encuentran formuladas en su forma original, sin adaptar su contenido al marco jurídico de destino. De este modo, contrariamente a los procedimientos llamados de localización o adaptación textual, el texto que presenta el EULA analizado ha sido traducido siguiendo parámetros de gran apego formal, sin considerar la posibilidad de que pueda ser considerado nulo dentro del ordenamiento español, de conformidad con lo dispuesto, entre otras, por la mencionada Ley General para la Defensa de los Consumidores. Cabe suponer, a este respecto, que el mero hecho de que el original esté siempre presente junto al texto traducido o se pueda acceder al mismo con facilidad escogiendo, por ejemplo, la instalación del software en un idioma u otro o consultando la página web del programa, se erige como un poderoso condicionante que determina la forma final de la traducción.

\section{Consideraciones discursivas sobre los End User License Agreements y sus equivalentes Contratos de licencia de usuario final}

Pasando de un enfoque jurídico a otro meramente lingüístico, encontramos, en el marco de la comunicación del discurso, que el texto se encuentra dirigido a todo aquel que pretenda hacer uso del software, identificado y referido de forma directa por el proveedor como el «usuario»:

NOTICE TO USER: PLEASE READ THIS CONTRACT CAREFULLY. BY USING ALL OR ANY PORTION OF THE ADOBE READER SOFTWARE ("SOFTWARE") YOU ACCEPT ALL THE TERMS AND CONDITIONS OF THIS AGREEMENT, [...]

AVISO PARA EL USUARIO: LEA CUIDADOSAMENTE ESTE CONTRATO. AL UTILIZAR EL SOFTWARE ADOBE READER ("SOFTWARE") O UNA PARTE DEL MISMO, USTED ACEPTA TODOS LOS TÉRMINOS Y CONDICIONES DE ESTE CONTRATO, $[\ldots]$

Del mismo modo, con las salvedades y limitaciones vistas anteriormente, tanto el tipo de servicio proveedor-usuario como la relación contractual sobre la que versa el objeto de la licencia se encuentra recogida en el marco jurídico español, si bien, ante un eventual litigio sobre la licencia, el proveedor del software analizado exige la incoación de procesos en tribunales muy probablemente distintos a los correspondientes a la jurisdicción del usuario de lengua castellana:

Governing Law. This Agreement will be governed by and construed in accordance with the substantive laws in force: (a) in the State of California, if a license to the Software is obtained when you are in the United States, Canada, or Mexico; or (b) 
in Japan, if a license to the Software is obtained when you are in Japan, China, Korea, [...]; or (c) England, if a license to the Software is purchased when you are in any other jurisdiction not described above.

Ley Aplicable. Este Contrato se regirá e interpretará de conformidad con las leyes substantivas vigentes en: (a) el estado de California, si se ha adquirido una licencia para el Software cuando se encuentre en los Estados Unidos de América, Canadá, o México; o (b) Japón, si se ha adquirido una licencia para el Software cuando se encuentre en Japón, República de China, República de Corea [...]; o (c) Inglaterra, si usted ha adquirido una licencia para el Software mientras se encontraba en cualquier otro país no descrito anteriormente.

Igualmente, son numerosos los indicadores que dan buena cuenta de la no originalidad del texto en lengua castellana y que llevan al lector a concebir, de forma implícita, el CLUF como una mera traducción. Aunque ya hemos mencionado que no se da en la licencia de uso en el presente estudio, en ocasiones se incluyen cláusulas que refieren a la lengua inglesa para la resolución de eventuales litigios, dado que el proveedor de software suele redactar la licencia en este idioma para posteriormente encargar traducciones multilingües que pretenden cubrir la mayoría de los mercados en los que se comercializa el software en cuestión. El proveedor, de este modo, se protege de las posibles reclamaciones que pudieran surgir a resultas de distintos errores de traducción.

\subsection{Uso de títulos y encabezados}

Uno de los indicadores más evidentes de la no redacción original del CLUF en lengua castellana es la presencia de títulos y encabezados en sus distintas cláusulas:

4. Restrictions.

4.1 Notices. You shall not copy the Software except as set forth in Section 2. Any copy of the Software that you make must contain the same copyright and other proprietary notices that appear on or in the Software.

4. Restricciones.

4.1 Avisos. Usted no podrá copiar el Software, excepto en los términos previstos en la Sección 2. Cualquier copia del Software que Usted realice deberá contener los mismos avisos de derechos de autor y de propiedad que aparecen en el Software.

La normativa de la modalidad textual de los contratos españoles no prevé la inclusión de títulos a modo de avanzadilla de cuanto disponen a su continuación; bien es sabido, por el contrario, el gusto de los textos ingleses por este tipo de fórmulas endofóricas que facilitan en gran medida la ordenación textual y permiten que la referencia a los distintos apartados se realice de forma más clara y precisa, bien por la campaña iniciada por la Plain English Campaing (Alcaraz, 2000: 46) en pos de un inglés más claro y accesible, bien por ser procedimiento de evidente costumbre.

No obstante, aunque no se observa en los documentos analizados, numerosos EULA y sus correspondientes CLUF incluyen una disposición en la que informan 
del carácter orientativo de los encabezados de las cláusulas, advirtiendo que el espíritu de lo estipulado se encuentra contenido en el párrafo en cuestión, y que el objeto de los títulos es, como ya hemos mencionado, facilitar la lectura a lo largo del documento, sin que éstos puedan ser argüidos como recurso en caso de litigio entre las partes.

En cualquier caso, si bien su inclusión no es vinculante y resulta extraña en la forma habitual de los contratos redactados en lengua castellana, encontramos a lo largo de todo el CLUF analizado la aparición de encabezados que no hacen sino ahondar en la idea de texto traducido condicionado por la presencia de tales elementos en el texto redactado en su lengua original.

\subsection{Calcos contrarios a la tipología textual jurídico-administrativa española}

De forma similar, se observan numerosos calcos léxicos y gramaticales contrarios a la normativa jurídico-administrativa española. De entre todos los calcos presentes en la traducción analizada, merece especial atención, por lo reiterado de su uso, el empleo del artículo demostrativo «este», con sus correspondientes variantes de género y número, que viene a sustituir, tanto en la traducción analizada como en la mayoría de los CLUF observados, lo que en el lenguaje jurídico-administrativo castellano se encuentra representado por el sintagma «el presente»:

10. General Provisions. If any part of this Agreement is found void and unenforceable, it will not affect the validity of the balance of this Agreement, which shall remain valid and enforceable according to its terms. This Agreement shall not prejudice the statutory rights of any party dealing as a consumer. This Agreement may only be modified by a writing signed by an authorized officer of Adobe.

10. Disposiciones Generales. Si se determina que cualquier parte de este Contrato es nula y no exigible, lo anterior no afectará la validez del resto del presente Contrato, que permanecerá válido y exigible de conformidad con sus términos. Este Contrato no perjudicará los derechos conferidos en las leyes de cualquier parte que opere como consumidor. Este Contrato sólo se podrá modificar mediante un escrito firmado por un representante autorizado de Adobe.

Asimismo, indicio inequívoco de su carácter no original es la presencia constante de estructuras y expresiones del todo inusitadas en lengua castellana:

1. Definitions. "Software" means (a) all of the contents of the files, disk(s), CDROM(s), or other media with which this Agreement is provided, including but not limited to (i) Adobe or third-party computer information or software; (ii) related explanatory written materials or files ("Documentation")

1. Definiciones. "Software" significa (a) todo el contenido de los archivos, disco(s), disco(s) CD-ROM o demás medios con los cuales este Contrato se adjunta, incluyendo, sin limitación (i) información computacional o programas de software de Adobe o de terceros; (ii) material escrito o archivos con información relacionada ("Documentación") 
Por ejemplo, en la primera cláusula de la licencia de usuario analizada, observamos la cantidad de calcos léxicos y gramaticales que redundan en la idea mencionada. En primer lugar, encontramos el empleo del vocablo «significar» en sustitución del verbo inglés «to mean». Sin embargo, teniendo en cuenta el género textual en el que se enmarca la traducción, sería más correcto el uso de la locución «por "Software" se entiende» en lugar de «"Software" significa».

Por otra parte, se observan expresiones poco frecuentes dentro de los textos jurídico-administrativos como «con los cuales este contrato se adjunta»o «sin limitación»: los elementos de estas oraciones no resultan en absoluto naturales, pudiéndose apreciar que en ambos casos se ha seguido una traducción excesivamente literal y condicionada por el EULA original, al tiempo que en lengua castellana encontramos locuciones del tipo «que acompañan al presente contrato» y «a título orientativo», respectivamente, mucho más frecuentes dentro de la tipología textual analizada. A este respecto, se aprecia también un error en el análisis de los sintagmas de la oración «Adobe or third-party computer information or software», ya que se ha traducido «computer» como si únicamente modificara a «information», cuando en realidad modifica tanto a este término como a «software». En este caso, la traducción debería haber sido, por tanto, «programas de software de Adobe o de terceros, o información sobre los mismos», y no «información computacional o programas de software de Adobe o de terceros».

\subsection{Uso no normativo de mayúsculas}

Bien es sabido que, en ocasiones, lo normativo ve peligrar su existencia ante el surgimiento de elementos novedosos, considerados en un primer momento incorrectos, pero que van ganando terreno y adeptos hasta fijarse de forma efectiva y eficiente dentro del sistema lingüístico y recibir la consideración de normativos. Tales elementos ven su origen en la multitud de ámbitos de uso de la lengua, como fiel reflejo de la ordenación del pensamiento social, y son sus hablantes quienes deciden incorporarlos finalmente a su idioma o rechazarlos por su carácter no normativo. Los textos traducidos suponen una de las principales vías de incorporación para los mencionados elementos. Bien por la necesidad de otorgar significantes novedosos a significados inexistentes en el sistema lingüístico de llegada, bien por comportamientos traductológicos involuntarios, se incorporan a la lengua castellana infinidad de términos que, si bien resultan extraños en un primer momento, su uso continuado posibilita su natural incorporación a la competencia lingüística de cada hablante.

En esta línea, en los últimos años se ha hecho más frecuente el empleo de mayúsculas en lengua castellana, fruto evidente del contacto con la lengua inglesa. Por ello, pese a que el español reserva el uso de mayúsculas a los nombres propios y a aquellas palabras localizadas al principio de una oración (Cascón Martín, 1999), es frecuente ver referencias bibliográficas o títulos en mayúsculas, a resultas de un calco evidente de la normativa inglesa. De este modo, detectamos en el texto traducido la presencia de mayúsculas que contravienen la normativa explicada y que se 
corresponden exactamente en número y posición con aquellas palabras que aparecen en mayúscula en lengua inglesa:

13.3 Prerelease Product Additional Terms. If the product you have received with this license is precommercial release or beta Software ("Prerelease Software"), then the following Section applies. To the extent that any provision in this Section is in conflict with any other term or condition in this Agreement, this Section $[\ldots]$

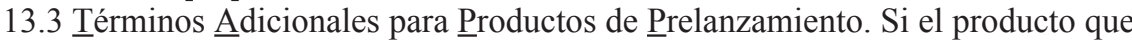

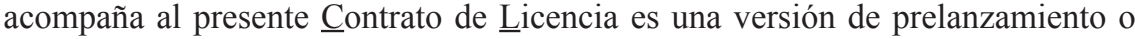

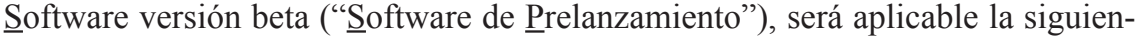

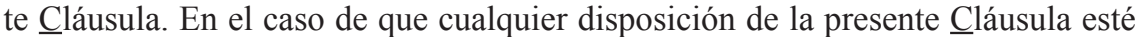
en conflicto con cualquier otro término o disposición del presente Contrato, lo dispuesto en esta $\underline{\text { Cláusula }[\ldots]}$

\subsection{Presencia de terminología técnica novedosa}

En línea con lo explicado, los sistemas lingüísticos poseen diversos mecanismos de creación semántica que surgen en aquellos casos en los que se pretende transmitir un significado de una lengua a otra y en los que la lengua receptora no posee un significante válido, bien por el carácter novedoso del término importado o bien como consecuencia de una extensión en el significado del mismo que impide la coincidencia terminológica de ambos significantes. El inglés, lengua más avanzada técnicamente que el español como lengua de uso en las comunidades en las que un gran número de productos y procesos tecnológicos ven la luz, así como en cuanto a su aceptado papel de lingua franca en la actualidad, genera una ingente cantidad de términos de inclusión problemática en la lengua castellana:

You acknowledge that the Software is a prerelease version, [...], and may contain bugs, errors, and other problems that could cause system or other failures and data loss.

Usted reconoce que el Software es una versión de prelanzamiento, [...] y que puede contener virus, errores y otros problemas que podrían causar fallos en el sistema o de otro tipo y pérdida de datos.

Unless otherwise expressly permitted hereunder, no other server or network use of the Software is permitted, including but not limited to use of the Software (i) either directly or through commands, data, or instructions from or to another computer or (ii) for internal network, Internet, or web hosting services.

Salvo disposición contraria establecida en este Contrato, no se permite el uso de otros servidores o redes, incluido pero no limitado al uso del Software (i) directamente o a través de comandos, datos o instrucciones a o desde otro equipo o (ii) para redes internas, $\underline{\text { Internet o servicios de sitios de red. }}$

Como se observa en los párrafos anteriores, la presencia de terminología especializada plantea cierta dificultad para la labor del traductor. Por lo general, la terminología informática ha hecho desde un primer momento uso de préstamos 
lingüísticos, como «software»o «phishing», lo que ha originado la presencia de infinidad de dobletes semánticos derivados de la traducción de tales préstamos, como es el caso de «programas informáticos» o «suplantación de identidad», por seguir el ejemplo. La presencia de estos elementos en el texto de partida supone un fuerte condicionante de traducción, ya que en algunos casos, sin que haya ninguna regla aparente al respecto, se opta por la utilización del término inglés («router», «hardware», etc.), mientras que en otros resulta del todo indiferente la utilización de un término u otro («email»o «correo electrónico», «software»o «programa informático», etc.) o bien se prefiere el término castellano («navegador» y no «browser», por ejemplo). En estos casos, serán los distintos condicionantes, como el grado de especialización del receptor de la traducción o las indicaciones dadas por el iniciador de la misma, los que determinen el empleo de una técnica u otra, si bien el traductor deberá ser coherente con los criterios adoptados a lo largo de todo el texto.

En ocasiones llama poderosamente la atención la escasa calidad de este tipo de textos, achacables en principio a dos factores: por una parte, una cierta impericia traductora frente a la tipología textual objeto de estudio; por otra, las exigencias del iniciador, que, sabido el carácter semi-implícito de la traducción y el fácil acceso al texto original, junto con una concepción del concepto de traducción en ocasiones errónea, hace prevalecer el apego formal y de contenido frente a las convenciones de la tipología textual de destino. Como ejemplo de ello, apuntaremos que, en el primero de los dos fragmentos anteriores, aparece en inglés el término «bug», traducido al castellano como «virus». No obstante, el mencionado término no hace referencia en realidad a ningún tipo de virus informático, sino que se acompaña en calidad de sinónimo a «errors» y se engloba en lo que el fabricante ha denominado «other problems», siendo su traducción más adecuada la de «error (o defecto) de programación».

En segundo lugar, resulta evidente que tanto por la ordenación sintáctica de la oración «Salvo disposición contraria establecida en este Contrato [...] para redes internas, Internet o servicios de sitios de red.» como por la formulación de sus estructuras léxicas, los significados contenidos en la misma resultan de difícil comprensión para el lector castellano, que seguramente apreciará los errores contenidos y redundará en la idea de que se encuentra ante un texto traducido. Y aunque no sea el propósito del presente estudio, se antoja necesaria la reformulación en castellano del citado segundo ejemplo, para dar buena cuenta de tal incorrección. Se propone, por tanto, el siguiente texto: «Salvo disposición contraria recogida en el presente contrato, no se permite el uso del software a través de redes ni de servidores, ni (i) la ejecución de órdenes, datos o instrucciones relativas al mismo realizada de forma directa o desde otro ordenador, ni (ii) su uso para redes internas, Internet o servicios de alojamiento web», que, si bien resulta mucho menos literal que la traducción analizada, conviene el significado del original de forma más clara y efectiva. 


\subsection{Errores de apreciación terminológica}

Tal y como se ha explicado, encontramos numerosos errores en la terminología traducida a lo largo del documento. Dada la naturaleza del presente estudio, citaremos dos de ellos de forma sucinta:

2.2.2 For information on how to distribute the Software on tangible media or through an internal network, refer to the section entitled "How to Distribute Adobe Reader software" at http://www.adobe.com/products/acrobat/distribute.html.

2.2.2 Para obtener más información sobre cómo distribuir el Software en un medio tangible o a través de una red interna, consulte las secciones bajo el título "Cómo Distribuir Adobe Reader" en http://www.adobe.es/products/acrobat /distribute.html.

You shall not reverse engineer, decompile, disassemble, or otherwise attempt to discover the source code of the Software except to the extent you may be expressly permitted to decompile under applicable law, $[\ldots]$

Usted no podrá utilizar la ingeniería inversa, descompilar, desmontar o de cualquier forma tratar de descubrir el código de origen del Software excepto en la medida en que esté expresamente autorizado a descompilar bajo la ley vigente, $[\ldots]$

Por una parte, se hace evidente la cantidad de préstamos de la lengua inglesa resultantes en neologismos cuanto menos extraños en lengua castellana, fruto quizá de la denominada «tentación paronímica» (Alcaraz, 2002), es decir, la atracción que despiertan las palabras homófonas y homógrafas y las construcciones sintácticas paralelas de la lengua de partida sin que su empleo sea sometido a análisis crítico. De este modo, la idea expresada en inglés mediante el sintagma verbal «reverse engineer, decompile, disassemble» encuentra su equivalente en español en el desmontar algo para averiguar su origen o funcionamiento. Por tanto, términos o expresiones como los empleados en la traducción, «utilizar la ingeniería inversa» o «descompilar», resultan superfluos y vanos para el lector castellano y su significado podría haber quedado recogido de forma mucho más natural simplemente mediante el empleo del verbo desmontar: «Usted no podrá desmontar el software ni tratará de ningún otro modo de descubrir su código fuente $[\ldots]$ ».

Por otra, tal y como se aprecia en la corrección propuesta, encontramos ulteriores errores en la terminología empleada. Los equivalentes castellanos de «source code» $\mathrm{y}$ «tangible media» son, respectivamente «código fuente» y «soporte físico»; en ningún caso «código de origen» o «medio tangible».

\subsection{Empleo de preposiciones dobles}

Ya se ha apuntado anteriormente que son abundantes los indicadores textuales que apuntan de forma inequívoca hacia la naturaleza del CLUF como texto traducido. Por una parte, encontramos elementos traducidos de forma distinta a las con- 
venciones del texto meta; por otra, elementos de difícil acomodo en lengua castellana, aunque cuya inclusión se antoja necesaria en el texto traducido de acuerdo con las pautas de apego formal al documento original adoptadas.

En el primero de los casos se incluye, junto con la mayoría de elementos textuales vistos hasta el momento, la presencia de preposiciones dobles en lengua inglesa. La aparición de estos elementos en el EULA analizado ha sido trasladada en la mayoría de ocasiones de forma literal al CLUF, lo que se muestra contrario a las convenciones de estilo de la lengua castellana, que, ante la presencia de dos tipos de preposiciones distintas referidas a un mismo sustantivo, hace uso de la fórmula PREPOSICIÓN1 + SINTAGMA NOMINAL + PREPOSICIÓN2 + EL/LA/LOS/LAS MISMO/A/OS/AS, frente a la fórmula PREPOSICIÓN1 + PREPOSICIÓN2 + SINTAGMA NOMINAL típica del inglés:

[...] Adobe may not introduce a product similar to or compatible with the Prerelease Software.

[...] Adobe no podrá introducir un producto similar $\underline{\mathrm{a}}$, o compatible $\underline{\text { con, }}$, el Software de Prelanzamiento.

De este modo, el ejemplo presentado habría estado más cerca de las convenciones textuales del español de haber sido traducido por «Adobe no podrá introducir ningún producto similar al software de prelanzamiento ni compatible con el mismo».

\subsection{Presencia de dobletes jurídicos extraños}

Por otra parte, en lo referente a los elementos de difícil acomodo en lengua castellana, encontramos la inclusión de dobletes jurídicos de carácter redundante sin equivalente en las convenciones textuales del texto de llegada. Dicho sea con otras palabras, en el lenguaje jurídico existen dobletes (e incluso tripletes) como «last will and testament» que pueden ser traducidos empleando un solo término castellano, mientras que otros como «all and sundry» necesitan de soluciones semánticas que distan del empleo de dobletes en castellano. No obstante, como consecuencia de los condicionantes de traducción anteriormente referidos, en el texto analizado aparecen dobletes poco habituales como «se regirá e interpretará» o «nula y no exigible», que inevitablemente pasan a aumentar la lista de elementos indicadores del carácter del CLUF como texto traducido vistos hasta el momento:

9. Governing Law.This Agreement will be governed by and construed in accordance with the substantive laws in force:

9. Ley Aplicable. Este Contrato se regirá e interpretará de conformidad con las leyes substantivas vigentes en:

10. General Provisions. If any part of this Agreement is found void and unenforceable, it will not affect the validity $[\ldots]$

10. Disposiciones Generales. Si se determina que cualquier parte de este Contrato es nula y no exigible, lo anterior no afectará la validez [...] 


\subsection{Presencia de siglas y simbolos textuales inexistentes en lengua castellana}

Uno de los aspectos textuales que más poderosamente llama la atención dentro del CLUF, y que con mayor fuerza incide en la idea de éste como texto traducido, es la presencia de siglas y símbolos inexistentes en lengua castellana, así como las menciones directas a la lengua inglesa referidas en el siguiente subapartado:

11. Notice to U.S. Government End Users. The Software and Documentation are "Commercial Items," as that term is defined at 48 C.F.R. $\$ 2.101$, consisting of "Commercial Computer Software" and "Commercial Computer Software Documentation,” as such terms are used in 48 C.F.R. $\$ 12.212$ or 48 C.F.R. $\$ 227.7202$, as applicable.

11. Notificación a los Usuarios Finales del Gobierno de los EE.UU. El Software y la Documentación constituyen "Elementos Comerciales" (Commercial Items), tal y como se define dicho término en el 48 C.F.R. $\$ 2.101$, consistente en un "Programa Informático Comercial" y "Documentación del Programa Informático Comercial", tal y como se utilizan dichos términos en el 48 C.F.R. $\$ 12.212 \mathrm{o}$ el 48 C.F.R. $\$ 227.7202$, según corresponda.

Encontramos en el fragmento analizado el acrónimo C.F.R. (Code of Federal Regulations), cuyo significado no ha sido explicado mediante la reformulación de sus siglas en castellano ni mediante ningún tipo de aclaración al lector. Sorprendentemente, en el mismo párrafo sí aparece entre paréntesis el término «Comercial Items» acompañando a su traducción en castellano, cuya comprensión no encierra ningún tipo de duda y cuya inclusión resulta radicalmente contraria a los procedimientos de traducción empleados en el texto analizado hasta tal momento. Igualmente, aparece en el CLUF el símbolo «§» desprovisto de cualquier referencia que indique su verdadero significado. La presencia de este símbolo se erige para numerosos traductores como un importante condicionante y suscita un intenso debate sobre la legitimidad de traducir siglas referentes a disposiciones legales, que, en cualquier caso, deberán ser identificadas en su lengua original si se desea acudir al ordenamiento legal en el que se originaron en caso de requerirse cualquier comprobación. En cualquier caso, si bien consideramos pertinente la inclusión en el CLUF del mencionado acrónimo y de cuantos otros participen del mismo carácter (aunque quizá resulte más clarificador para el lector del texto meta que sus siglas se desarrollen traducidas entre paréntesis), el símbolo «§» provoca demasiada extrañeza en lengua castellana, por lo que debería haberse incluido «párrafo» en su sustitución en cada una de las ocasiones en las que aparece en el texto traducido.

\subsection{Mención directa a la lengua inglesa}

Encontramos por último una referencia directa a la lengua inglesa: «por su sigla en inglés». Si bien el objeto de tal aclaración, prescindible, por otra parte, es la de facilitar la comprensión al lector del texto, la idea que transmite, esta vez de forma 
evidente, no es otra que la apuntada a lo largo del presente estudio: la naturaleza del CLUF como texto no original, surgido a partir de un texto ajeno a la cultura receptora, en este caso el EULA, y de su traducción, sometida a los múltiples condicionantes analizados, en lengua castellana.

[...] for the sole and exclusive purpose of (a) using the Software (from an unlimited number of client computers on your internal network) via (i) the Network File System (NFS) for UNIX versions of the Software or (ii) Windows Terminal Services $[\ldots]$

[...] para el único y exclusivo fin de utilizar el Software mediante (a) el Sistema de Archivos de Red (NFS, por su sigla en inglés) para versiones UNIX del Software o (b) Servicios de Terminales Windows [...]

\subsection{Anisomorfismo jurídico}

Como se ha venido apuntando hasta el momento, tales condicionantes presentan una seria dificultad en el proceso traductor. La necesidad de dar cabida a la totalidad de términos jurídicos que aparecen en lengua inglesa, atendiendo al mismo tiempo a los condicionantes de traducción anteriormente mencionados, se refleja en el CLUF analizado a través de la presencia de términos con significados carentes de sentido dentro de la tipología textual castellana en la que se engloban. De este modo, tal y como se puede observar en los siguientes fragmentos del texto analizado, aparecen calificativos como «incidental», «especial», «establecido por ley» o «ejemplar» que no se encuentran recogidos ni por la lengua castellana ni por el ordenamiento jurídico español como posibles modificadores del sustantivo «daño», así como sintagmas referidos a «condiciones razonables», que se suponen no equivalentes a «reasonable conditions», puesto que lo razonable se mide indudablemente desde el ordenamiento jurídico en cuestión, y aquello considerado como razonable en el ordenamiento estadounidense, bajo cuya jurisdicción se redactó el documento EULA analizado, puede que no se considere como tal en el ordenamiento español y en los distintos países de habla hispana en los que se comercializa el software.

[...] ANY DIRECT, INDIRECT, INCIDENTAL, SPECIAL, STATUTORY, PUNITIVE, EXEMPLARY, OR CONSEQUENTIAL DAMAGES WHATSOEVER $[\ldots]$

[...] CUALQUIER DAÑO DIRECTO, INDIRECTO, INCIDENTAL, ESPECIAL, ESTABLECIDO POR LEY, PUNITIVO, EJEMPLAR O EVENTUAL [...]

EXCEPT FOR ANY WARRANTY, CONDITION, REPRESENTATION, OR TERM TO THE EXTENT TO WHICH THE SAME [...]

EXCEPTO POR CUALQUIER GARANTÍA, CONDICIÓN, REPRESENTACIÓN O TÉRMINO, EN LA MEDIDA EN QUE LOS MISMOS [...]

Adobe has the right to impose reasonable conditions and to request a reasonable fee before providing such information. 
Adobe tiene el derecho de imponer condiciones razonables y de solicitar el pago de una cuota razonable antes de proporcionarle dicha información.

\subsection{Transposiciones gramaticales}

Finalmente, cabe señalar, como condicionante del aspecto final de la traducción del EULA al castellano, el carácter de la propia gramática inglesa y la necesidad de acomodar sus elementos morfosintácticos a la gramática española. Según López Guix y Wilkinson (1999:123), la «simplicidad morfológica» de los verbos ingleses «fuerza la mano del traductor» redundando en el uso de transposiciones y otros cambios. De acuerdo con ello, resulta necesario incluir en el texto traducido las posibles acepciones de las formas gerundivas inglesas, capaces de alternar las categorías de verbo, sustantivo y adjetivo, con el fin de cubrir la totalidad de las interpretaciones legales que de su lectura se pudieran derivar. De tal modo, como se muestra a continuación, se observa la presencia de los términos «uso», «usado» y «utilización», en sustitución de «use» y «using»:

"Use" or "Using" means to access, install, download, copy, or otherwise benefit from using the functionality of the Software in accordance with the Documentation. Los términos "Uso", "Usado" o "Utilización" significan acceder, instalar, descargar, copiar o de cualquier otra forma obtener beneficios de la utilización de las funciones del Software de acuerdo con la Documentación.

\section{Conclusiones}

Una vez realizado el análisis de la licencia de uso propuesta, resulta especialmente destacable el cuantioso número de elementos léxicosintácticos propios de la lengua inglesa que aparecen en su traducción al castellano. Este hecho, junto con la presencia de los fuertes condicionantes de traducción hallados, confiere al CLUF una naturaleza particular y diferente a la propia de la tipología textual en la que se enmarca. De forma general, se puede afirmar, de acuerdo con lo observado a lo largo del presente estudio, que la naturaleza de los CLUF guarda una gran fidelidad formal y estructural a los EULA a partir de los cuales vieron la luz, tanto a nivel macro como microestructural, cayendo en numerosas ocasiones en la comentada «tentación paronímica».

En el primero de estos niveles, se ha comprobado que la fidelidad textual del texto traducido al original es máxima, aproximándose en ocasiones de forma muy cercana a la invalidez del mismo como instrumento jurídico. Tal y como resulta del análisis llevado a cabo, algunas de las aseveraciones contenidas en el documento original se antojan válidas dentro del ordenamiento jurídico desde el que surgen, pero su inclusión en el texto traducido contraviene en ocasiones el ordenamiento español en general y las distintas disposiciones relativas a los derechos de los usuarios y al carácter abusivo de ciertas cláusulas en particular. 
En el plano microestructural, también se ha puesto de manifiesto una excesiva vinculación del documento traducido con su original, tanto a un nivel meramente textual, en lo relativo a la numeración de las cláusulas del contrato, a la ordenación y disposición de párrafos o a las normas de puntuación, como en un estadio léxico, en el que se ha mostrado la utilización de una terminología en ocasiones extraña e irrelevante que se presupone contraria a las expectativas textuales del lector de la traducción analizada.

Como es bien sabido, la presencia de condicionantes es un poderoso modulador del aspecto final del texto traducido. El anisomorfismo de los marcos jurídicos de origen y de llegada, así como de muchos de los conceptos que encierra el documento original, es innegablemente uno de ellos. En cualquier caso, su impacto debería quedar minimizado con los mecanismos de traducción pertinentes que permitan la adecuación del documento en cuestión a la tipología textual a la que se adscribe. Así pues, la traducción ha de resultar textual y funcionalmente válida en comparación con aquellos documentos de idéntica índole redactados originariamente en la lengua de llegada, rechazando aquellos elementos que difieran de los habituales en el marco lingüístico empleado.

De lo contrario, ante la constante presencia de acepciones y estructuras encontradas en el texto analizado ajenas a la lengua castellana en general y a la tipología jurídico-administrativa española en particular, el lector podrá intuir la naturaleza real del mismo, suponiendo de forma acertada su creación a partir de un original redactado en lengua extranjera, lo que, si bien en ocasiones no impide su validez un cuanto a instrumento jurídico, pone de relieve la falta de adecuación con las convenciones textuales del mismo, menoscabando muy probablemente de forma subjetiva la validez del texto como instrumento jurídico. Por tanto, como ya se ha mencionado, cabe proponer, en pos de tal acomodo textual y validez efectiva de este tipo de traducciones, un mayor esmero y atención a la hora de valorar y adecuar los distintos tipos de condicionantes de traducción a los que se pueda ver sujeto el texto de llegada, una más precisa elección de las estructuras léxicolingüísticas empleadas y una particular atención a las disposiciones vigentes en España respecto al marco jurídico-normativo en cuestión. Como no puede ser de otra manera, dicha labor ha de recaer tanto sobre la pericia del traductor y su capacidad para localizar aquellos elementos de difícil incursión en el texto de destino como del iniciador de la traducción, que ha de tomar mayor conciencia de la importancia de los contratos de licencia de usuario final como instrumento jurídico en la cultura receptora.

\section{Referencias bibliográficas}

Alcaraz Varó, E., El inglés jurídico. Barcelona: Ariel 1994.

Alcaraz Varó, E., El inglés profesional y académico. Madrid: Alianza 2000.

Alcaraz Varó, E., El español jurídico. Barcelona: Ariel 2002.

Alcaraz Varó, E. et al., El inglés jurídico norteamericano. Barcelona: Ariel 2001.

Alcaraz VARó, E. et al. (eds.), Las lenguas profesionales y académicas. Barcelona: Ariel 2007. 
Alcaraz Varó, E. / Hughes, B., Diccionario de términos jurídicos, Inglés-Español, Spanish-English. Barcelona: Ariel 1993.

BAASE, S., «IBM: producer or predator?», Reason (1974), 4-10.

BAIn, M. et al., Aspectos legales y de explotación del software libre. Barcelona: Universitat Oberta de Catalunya 2004.

CAscón Martín, E., Manual del buen uso del español. Madrid: Castalia 1999.

GÉMAR, J.C., «La asimetría cultural y el traductor jurídico. El lenguaje del derecho, la cultura y la traducción», en: Monzó NeBot, E. / BorJA AlBi, A. (eds.), La traducción y la interpretación en las relaciones jurídicas internacionales. Castellón: Universidad Jaume I 2005, 34-64.

Gómez Perals, M., La cesión de uso de los programas de ordenador. Madrid: Colex, 1999.

LóPez GuIX, J.G. / WiLKInson J.M., Manual de traducción inglés-castellano: teoría y práctica. Barcelona: Gedisa 1997.

López-Tarruella Martínez, A., Contratos internacionales y de software. Valencia: Tirant lo Blanch 2006.

Martinez De Sousa, J., Diccionario de uso de las mayúsculas y minúsculas. Gisón: Trea 2007.

Martínez GARcíA, A., «Contrastes interculturales del inglés al español: singularidades léxicas del lenguaje jurídico con la ejemplificación de los términos 'barrister' y 'solicitor'», en: San Ginés Aguilar, P. / Ortega Arjonilla, E. (eds.), Introducción a la traducción jurídica y jurada (inglés-español). Granada: Comares 1996, 325-346.

Montero MARTínez, S. et al., "Condicionantes del proceso traductor: un caso de traductorexperto», Sendebar 12 (2001), 283-300.

Rico CARrillo, M., «Validez y regulación legal del documento y la contratación electrónica», Alfa Revista Electrónica de Derecho Informático 2000.

Collin, S.M.H., Spanish computing dictionary: Spanish-English, English-Spanish. Londres: Bloomsbury 2004.

VARGAS Sierra, C., «Algunas consideraciones prácticas sobre la traducción de un tipo de texto jurídico: el contrato de franquicia», Interlingüística 14 (2003), 1011-1026. 